\title{
Téoros
}

Revue de recherche en tourisme

\section{Le macro-inventaire du patrimoine}

Enfin, l'espoir de voir refleurir de beaux paysages

\section{Jean Désy}

Volume 5, numéro 1, mars 1986

Tourisme et régions

URI : https://id.erudit.org/iderudit/1080591ar

DOI : https://doi.org/10.7202/1080591ar

Aller au sommaire du numéro

Éditeur(s)

Université du Québec à Montréal

ISSN

0712-8657 (imprimé)

1923-2705 (numérique)

Découvrir la revue

\section{Citer cet article}

Désy, J. (1986). Le macro-inventaire du patrimoine : enfin, l'espoir de voir refleurir de beaux paysages. Téoros, 5(1), 26-27.

https://doi.org/10.7202/1080591ar d'utilisation que vous pouvez consulter en ligne.

https://apropos.erudit.org/fr/usagers/politique-dutilisation/ 


\section{Enfin, l'espoir de voir refleurir de beaux paysages Le macro-inventaire du patrimoine}

par Jean Désy*

\section{Une vaste opération de sauvetage...}

Voici, comme entree en matière, quelques points de repère de l'itinéraire récent du ministère des Affaires culturelles du Québec et qui a de quoi impressionner par ses résultats tangibles.

1972:

Adoption de la loi sur les biens culturels, qui consacre la nécessité de produire un inventaire "des biens culturels susceptibles d'être reconnus ou classês".

- Jean Desy est professeut de geographie au departe ment des sciences humaines de rUniversite du Qué bec a Chicoutimi.
1974:

Création de la Direction générale du patrimoine. Point de départ d'un programme d'inventaire élargi (architecture, ethnologie, oeuvres d'art archéologie)

\section{7:}

Déclenchement du programme de macroinventaire conçu comme outil d"inventaire, d'analyse, de synthese et de planification des lieu $x$ et des biens culturels de tout le territoire québécois, dégageant ainsi une vision globale de la richesse patrimoniale du Québec.

1983:

Début de l'exposition itinérante: " $L$ 'aménagement, l"urbanisme et le patrimoine"
1985:

Publication par le Service du patrimoine du $M A C$. de cet excellent guide géneral: Le macro-inventaire du patrimoine québécois. devancé de deux ans par un second. plus spécifique: L'anénagement, l'urbanisme et la patrimoine... une harmonie nouvelle à rechercher"l!. Et bien sür. entre temps, consignation de 250,000 photographies dans 94 rapports destines aux MRC, des centaines de répertoires. fichiers, pré-inventaires, études el expertises, cartes à toutes échelles...!

Les quelques pages qui suivent tenteront de faire un tour rapide du nouvel instrument mis au point par le MAC et d'entrevoir le

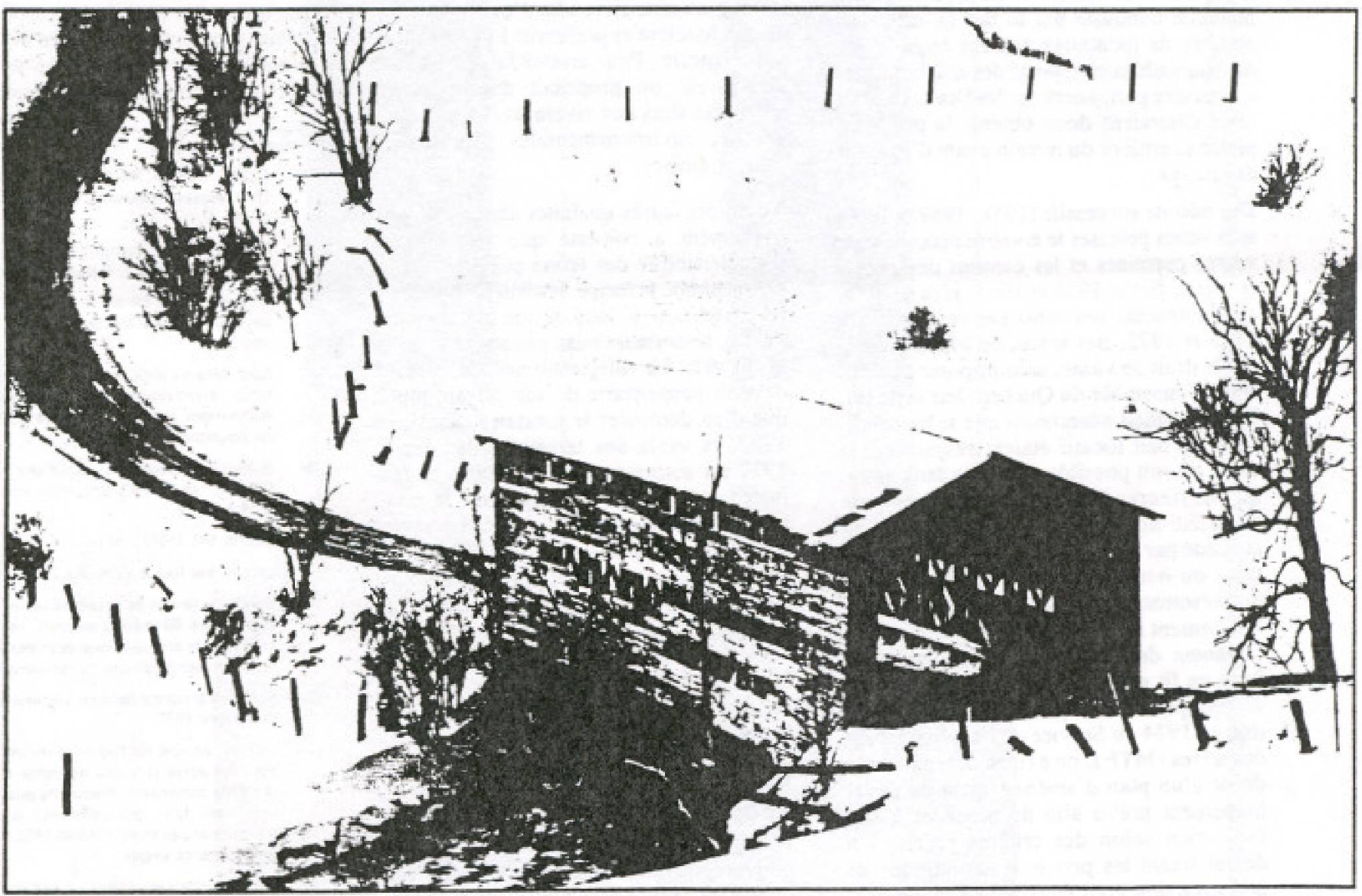

Le macro-inventaire, un instrument privilégiẻ de découverte, de protection et de mise en valeur du patrimoine. 
pourquoi et le pour qui de son existence, de même que le mode d'emploi possible.

De fait, le macro-inventaire comprend six volets: 1'histoire, l'archéologie, l'ethnologie, l'architecture et l'art religieux. I'inventaire et l'analyse du paysage architectural régional . mais aussi urbain (Montréal, Québec et Trois-Rivières). On a donc réussi, en l'espace de six ou sept ans, à identifier, localiser, photographier, ficher et cartographier l'ensemble des lieux et des biens culturels québécois d'une bonne valeur patrimoniale! Et cette information est accessible et actuelle. C'est dife en d'autres termes, que les intervenants touristiques, entre autres, disposent d'un outil inégalable d'évaluation d'une partie déterminante de l'offre touristique... ou qui devrait le devenir impérativement? Nous pouvons maintenant feuilleter à loisir l'album de famille du Québec pré-historiqué dans ses "anefacts" visibles, nous pouvons consulter le fichier des empreintes visibles et remarquables, dans le paysage, du Québécois en féte, en deuil, en prière, au travail ou retranché dans sa maison. Si ce n'est pas là une matière première essentielle au développement et à l'aménagement des régions touristiques, qu'est-ce donc alors?

\section{Notre patrimoine menacé de disparition}

Cette question nous amène à réfléchir trente secondes sur le pourquoi d'une telle entreprise. Allons-y sans détour: depuis une quarantaine d'années, le paysage architectural du Québec "fout le camp". Il suffit d'ouvrir un oeil le moindrement critique sur la dégradation scandaleuse du cadre băti de l'environnement, pour constater qu'au rythme où vont les choses, la laideur de l'habitat l'emportera sur la splendeur du paysage naturel! La moitié du Québec résidentiel en milieu rural s'est enveloppé de l'affreux "clapboard" d'aluminium ou de vinyle blanc, sept pouces de large, défigurant ainsi certains joyaux architecturaux. Le bungalow de banlieue s'installe en maitre dans tous les rangs. le mauvais goutt, le laisser-faire sinon la bettise règlent habituellement la poussée irrésistible des styles et des matériaux modernes, sans le moindre souci d'harmonie avec l'environnement et la tradition architecturale. Bref, cette charge un peu émotive ${ }^{|2|}$, donc a la limite caricaturale, contre les pratiques urbanistiques actuelles, veut mettre en relief l'urgence de déterrer nos vraies valeurs patrimoniales, de les mettre en lumière et de proposer au moderne de se marier dayantage à l'ancien. Pour le plus grand bien des régionaux, des régions qui se veulent touristiques et des touristes eux-mêmes.

Mais, plus précisément, de quelle manière le MAC propose-t-il d'utiliser ce macroinventaire? La seconde partie de leur récente publication suggère presqu'une douzaine de "récupérations" possibles. dont au moins une touche plus directement au tourisme, mais toutes indirectement:

- Sauvegarder, mettre en valeur et sensibiliser au patrimoine.

- Mieux cerner les régions culturelles et développer des liens d'appartenance.

- Élaborer des thèmes et des circuits d'interprétation.

- Prévenir et minimiser les impacts environnementaux de grands projets.

- Préparer des schémas régionaux d'aménagement.

- Préparer des plans et des règlements municipaux d'urbanisme.

- Mettre au point des programmes ou des projets speciaux de mise en valeur

- Esquisser des répertoires et des guides ayant trait à la restauration (de bătiments...).

- Faciliter l'attribution de statuts juridiques à des ensembles ou à des biens culturels.

- Faire un choix plus judicieux des études et des inventaires à venir.

- Susciter des perspectives intéressantes au plan de la recherche archéologique.

\section{Un outil pour reconnaître nos régions culturelles}

Signalons qu'à ce jour, plusieurs MRC et municipalités ont utilisé le macro-inventaire pour se conformer à l'article 11 de la loi 125 , stipulant " $/$ "identification des territoires présentant un inter rêt historique, culturel ou esthérique". Plusieurs guides de sensibilisation au patrimone se publient régulièrement ces derniers temps, témoignant souvent moins d'une préoccupation touristique prioritaire que d'un souci d'identification et d'appartenance régionale des habitants à leur territoire, dans une volonté de sensibilisation et de sauvegarde de notre héritage collectif ${ }^{(3)}$. Le Service du patrimoine suggère de dégager du nouvel outil, trois types d'itinéraires:

- Des circuits d'interprétation patrimoniale ayant pour theme et points d'attraction particuliers des phénomènes et des lieux d'intérét majeur à l'échelle des régions culturelles.

- Des parcours urbains plus réduits se prétant davantage à la circulation piétonne ou au transport en commun guidé

- Des parcours intégrés de certains circuits culturels aux grands réseaux touristiques existants. La série au 1:250,000 du MER/MICT et ATR. dans 18 régions touristiques du Québec, intitulee: Carte de tourisme et de plein air illustre déjá les possibilités de ce côté

\section{En conclusion}

Il nous apparait done que le macroinventaire du patrimoine québécois se présente comme un instrument privilégié de découverte, de protection et de mise en valeur de cette plus-que-ressource qu'est le patrimoine. Partie de l'environnement, il peut occasionnellement constituer tout l'environnement. L'outil s'adresse à de multiples intervenants régionaux situés à des points stratégiques de la "chaine parrimoniale "sélus et fonctionnaires des différents paliers de gouvernements, agences de voyage et bureaux de tourisme, promoteurs, constructeurs immobiliers, architectes et urbanistes, propriétaires de bátiments anciens, mais aussi et surtout le citoyenhabitant de son "pays" d'appartenance. Pour qu'il retrouve ses racines, retrouve la beauté d'un paysage architectural et qu'il ne contribue jamais plus à banaliser, défigurer et déshumaniser notre héritage commun, capital-nature comme "capitalen-murs. ".

\section{$+$}

\section{Notes et reforences}

(1) Gouvernement du Quebec. Le macro-inventaire du patrimoine quebereois. Quebec, Les Publica tions du Quebec. MAC, 1985, 150 pages.

L'amónagement. l'urbenisme at le patrimoine.. une harmonie nouvelle a rechercher. Quebec. MAC, 1989,79 pages.

121 Pour une démonstration plus complete sur ce sujet. voir un prochain article a paraitre dans la revue Tra. fic vol. 2, no. 7. (le magazine du Saguenay-Lac. St-Jeanl: De to misere des revéfements rési dentiels.

(3) MRC de Montmagny, Une fenetre sur notre his toire. Guide de sensib lisation au patrimoine de la MRC de Montmagny. Québec, 1985, 65 pages. MARTEL, Gaston. Guide ofu patrimoine cuiture touristique du Saguenay-Lac-St-Jean. Trafic. vol. 2. no. 2.3, 6te 85, p. 11.52 .

GAGNON, Gaston. Le patrimoine immobiliar de Chicoutimi Chicoutimi, service d urbanisme, Ville de Chicoutim, juin 1981, 78 p. + annexes. PROVENCHER, Jean, Le patrimoine agricole et horticole au Quebec. Québec. Commission des biens culturals du Oudbec, 1984, 94 pages. 